\title{
PATRyCJa Przelucka
}

Università della Slesia, Katowice

iD https://orcid.org/0000-0003-4157-2868

\section{La post-fantasy: \\ il ciclo letterario Inquisitore Eymerich di Valerio Evangelisti come una realizzazione dell'elemento fantastico tramite la narrazione postmoderna}

\author{
Post-Fantasy: \\ The literary series of Inquisitore Eymerich \\ by Valerio Evangelisti as an example of postmodern transposition \\ of the fantastic element
}

\begin{abstract}
This article examines the literary series of Inquisitore Eymerich by Valerio Evangelisti, a variegated macronarrative, which, resisting generic classification, introduces innovation to the panorama of contemporary Italian fantasy. Departing from the analysis of the salient features of the series such as intertextuality, hybridization, storiographic metafiction, and transfictionality, the article examines closely the areas of postmodern narration and inquires into the role of the fantastic element in Italian literature. Evangelisti makes the most of postmodern narrative strategies and of fantasy's ability to penetrate various media in order to provide a tool of critical reflection on human history with an emphasis on the phenomenology of power. By interweaving different literary genres and inviting readers to produce their own interpretations in form of fan fiction, Evangelisti creates a new type of committed narrative, post-fantasy, which presents a new conception of postmodern narrative, one that overcomes the barrier between the literary fiction and the genre fiction.
\end{abstract}

KeY Words: Italian fantasy, postmodern novel, intertextuality, transfictionality, narrative modes 


\section{Premessa}

A cominciare dal suo riconoscimento come termine letterario, il fantastico non ha cessato di imporsi all'attenzione della critica letteraria ed in Italia ha suscitato una produzione copiosa di scritti relativi all'argomento. Però, mentre gli studi letterari italiani hanno esaminato a fondo la letteratura fantastica fino alle opere di Italo Calvino, la considerazione accademica del fantastico dopo il 1970 non è stata abbondante. Molto spesso quei contributi che sono stati scritti tendono a non presentare le scritture fantastiche come tali ma piuttosto solo come fenomeni peculiari dei singoli autori. Inoltre, sono rarissimi gli studi dedicati al cosiddetto fantastico di genere, che in realtà ha avuto non poco rilievo nell'evoluzione della letteratura fantastica in Italia. D'estremo interesse è che nello stesso tempo siano stati scritti tantissimi articoli e saggi divulgativi sui giornali, sulle riviste e nelle antologie di narrativa che esaminano a fondo il fantastico italiano, non discriminando fra la letteratura colta e di genere e presentando una tradizione della letteratura fantastica italiana indipendente dai modelli occidentali. Allora ci si deve porre una domanda: qual è oggi il canone del fantastico italiano? Con quest'articolo si intende fornire una risposta a tale domanda e cercare di sopperire alla lacuna negli studi letterari italiani, analizzando una delle realizzazioni contemporanee più rappresentative del fantastico popolare, cioè il ciclo letterario Inquisitore Eymerich di Valerio Evangelisti, una macronarrazione composta da tredici volumi usciti dal 1994 al 2018, il cui primo volume ha vinto il Premio Urania.

Valerio Evangelisti ha riscosso un notevole successo presso il pubblico, diventando il punto di riferimento per gli altri autori della letteratura del genere ed indubbiamente ha creato una nuova qualità della fantasy, proponendo la sua versione postmoderna. Gli elementi fantastici nel postmodernismo sono comunemente accettati come componente costante della poetica postmoderna, ma la percezione della fantasy attraverso il prisma delle strutture di narrazione postmoderna è ancora una cosa rara (STASIEWICZ, 2016: 292). Il presente breve studio intende rivelare che le strategie della narrazione postmoderna ${ }^{1}$ adoperate da Evangelisti nel ciclo eymerichiano quali l'intertestualità, l'ibridazione dei

${ }^{1}$ Per l'analisi di alcuni aspetti della narrazione postmoderna italiana si veda CeSERANI (1997: 146-208). Secondo l'insigne studioso, il sistema della narrazione postmoderna è caratterizzato dai procedimenti seguenti: la dimensione oppressiva e foucaltiana del potere e dei limiti delle conoscenze umane, la rivisitazione del passato e della storia come consumo culturale e come affresco decorativo, l'intertestualità multilivello, la strategia retorica del pastiche, l'ibridazione di generi, il registro pluristilistico. Tra i temi adoperati dalla narrazione postmoderna si includono principalmente quelli del dubbio ontologico, del disorientamento spaziale o temporale, dei misteri, degli enigmi e del complotto. Si può anche constatare che la narrazione postmoderna italiana appartenga prevalentemente alla fenomenologia dell'interestualità. 
generi, la finzione metastorica e la transfinzionalità introducono l'innovazione nel panorama della fantasy italiana contemporanea, espandendo i confini e la funzione del genere.

Il ciclo racconta la storia di Nicolas Eymerich, domenicano e Inquisitore generale d'Aragona, un personaggio ispirato dall'uomo realmente vissuto, che cerca di purificare la terra dal male, dagli eretici e da diverse manifestazioni di Satana quali pagani, spettri, vampiri, zombie, posseduti, dischi volanti e minacce cibernetiche. L'intera opera, ambientata in continenti diversi, copre tre livelli temporali strettamente interconnessi: il Trecento (il cosiddetto tempo base) cioè la realtà storica dell'inquisitore, il livello 1 tra il XX e XXI secolo che costituisce più $\mathrm{o}$ meno la nostra contemporaneità e il livello 2 del futuro remoto che si estende anche molto oltre, per secoli, fino al XXXII secolo. Il discorso che è alla base del ciclo è la storia umana intesa come storia di conflitti e la storia del potere. Nel ciclo eymerichiano non esiste una verità assoluta, ma esiste una vasta gamma di verità, prospettive e interpretazioni. La realtà è un criterio opinabile ed è il potere che determina i rapporti tra gli uomini e il mondo. «Se il potere è forte, è esso a creare la realtà» (LipPI, 2000: 12). Evangelisti presenta con il ciclo Inquisitore Eymerich che la sua versione della fantasy non sia altro che un modo per trasfigurare e comprendere meglio gli incubi della storia moderna e passata.

\section{Universo inter- e intratestuale di Eymerich}

Analizziamo ora le caratteristiche postmoderne che distinguono il ciclo di Eymerich dalle narrazioni tradizionali conosciute dalla produzione di Tolkien ed i suoi imitatori. Cominciamo dalla caratteristica fondamentale che permea la narrazione postmoderna - l'intertestualità, un procedimento compositivo oppure retorico-discorsivo effettuato in forme diverse, che consiste nell'impiego massiccio di riferimenti ad altre opere ed anche agli stili e linguaggi diversi. Essendo alla base della riscrittura di testi, l'intertestualità è diventata il perno della creatività degli scrittori postmoderni italiani a partire da Italo Calvino fino ai postmodernisti consapevoli quali Umberto Eco, Antonio Tabucchi e Michele Mari. La sfrutta a fondo anche l'autore di Inquisitore Eymerich. Si possono distinguere tre piani sui quali si crea la complessa rete dell'intertestualità del ciclo. Sul primo piano, questo procedimento consiste nel dialogo con gli altri testi culturali, storici, religiosi e mitologici. Sul secondo piano, i romanzi compositivi del ciclo, in quanto prequel e sequel alternati, ed anche i livelli dentro un singolo volume dialogano infatti tra di loro nella maniera intratestuale ed autoreferenziale, nel circolo ermeneutico, espandendo ed integrando la conoscenza e l'interpretazione 
del lettore sul protagonista e sulla trama. Il terzo piano delle relazioni intertestuali consiste invece nell'ibridazione dei generi letterari.

Per quanto riguarda il primo piano, Inquisitore Eymerich spazia tra molteplici fonti d'ispirazione per fornire spunti narrativi per singole scene o per la costruzione di un mondo. Evangelisti si ispira agli stili degli altri autori come Lovecraft, Sheckley, Dumas, Salgari, Hammett, Souvestre e Allain (Tosoni, 2002), ma questo rimane implicito nelle scelte stilistiche e narrative, mentre le fonti scritte particolari sono spesso esplicitamente citate nel testo, sia dai personaggi che nella bibliografia finale in chiusura dei volumi. Il repertorio testuale di riferimento è molto ampio con le fonti disparate dalle modalità e funzioni diverse. Tra i rimandi espliciti troviamo monografie e documenti storici, agiografici e teologici, bolle pontificie, leggende italiane, manuali di magia, la Cabala giudaica, libri della ricerca alchemica, libri di negromanti, saggi di materie scientifiche (sia del XIV secolo che contemporanei), esposizioni di teorie, resoconti di esperimenti, articoli e inchieste di cronaca, notizie di attualità, opere paraletterarie, teorie del complotto e fonti di controinformazione. Poi ci sono citati dei testi sacri quali Bibbia, Vangelo, Lettere di San Paolo, Atti degli Apostoli, Apocalisse, che servono al protagonista come il punto di riferimento per argomentare e giustificare il suo sistema di convinzioni più salde. Inoltre, le opere dei Padri della Chiesa, da Origene a Sant'Ambrogio e San Tommaso d'Aquino, gli offrono le interpretazioni degli avvenimenti, mentre i testi letterari di autori classici, come Ovidio, Omero e Virgilio, di autori medievali e rinascimentali quali Dante e Petrarca ed anche di poeti contemporanei, fanno da indizi per risolvere gli enigmi messi nei libri (SEBASTIANi, 2018: 128).

Il secondo piano dell'intertestualità rivela legami profondi tra vicende, personaggi e questioni dei volumi diversi del ciclo che danno origine alla grandiosa architettura narrativa effettuata da Evangelisti da anni, composta e scomposta continuamente, frammentata e nascosta in volumi diversi attraverso le soluzioni linguistiche e strategie narrative ricorrenti che mettono in relazione $\mathrm{i}$ tredici romanzi tra di loro. Inoltre, i tre livelli temporali della trama hanno uno svolgimento autonomo, ma sono dipendenti l'uno dall'altro. Certamente, il sovrapporsi di piani temporali non è un'invenzione di Evangelisti, ma di maggior originalità qui è che i livelli si influenzano e chiarificano reciprocamente. L'essenza cardine dell'universo pluridimensionale creato da Evangelisti è costituita dalla teoria di psitroni formulata da Marcus Frullifer nel livello 1. Gli psitroni sono particelle «simili a neutrini, eccitate dall'attività cerebrale umana e proiettate da un cervello all'altro», capaci di viaggiare ad una velocità superiore a quella della luce (EVANGELISTI, 1994: 7). Lo stesso pensiero condiviso da molte persone eccita grazie all'attività delle loro sinapsi cerebrali gli psitroni, che come neutrini esistono intorno a noi, li carica con informazioni e gli dà una forma materiale. Se questo gruppo è assistito da una persona dalla spiccata attività della mente, un medium, il gruppo è in grado di mandare la forma tangibile del pensiero nello 
spazio e perfino nel tempo (135-137). Questo è il concetto più rilevante per la struttura e l'organizzazione della trama. Così, come vediamo nel primo volume del ciclo, è possibile realizzare nel livello 2 i viaggi interstellari ed i viaggi del tempo senza rischiare la vita degli astronauti perché l'astronave psitronica insieme con l'equipaggio è solamente una proiezione materiale dell'immaginario collettivo, una copia sacrificabile di esattamente la stessa nave ormeggiata al sicuro sull'orbita intorno alla Terra (135-137). Grazie allo stesso principio, nel tempo base le donne che credono ardentemente nell'avvento della dea Diana le danno un'evocazione materiale di una donna enorme. Questo caso è esemplare dell'interconnessione strettissima dei livelli temporali perché nello stesso giorno nel livello 1 Frullifer vuole dimostrare al pubblico la prova della sua teoria e fa apparire una visione materiale, avendo in testa Cynthia, una collega da cui è attratto. Siccome il nome Cynthia significa originario del monte Cinto, cioè un soprannome di Diana (225), l'evocazione della dea del tempo base ottiene la faccia di Cynthia. Ma grazie allo stratagemma dell'inquisitore Eymerich le donne invocano non Diana, ma Satana e lo stesso accade nel livello 1. Anche nel futuro remoto del livello 2, durante una spedizione cattolica sul pianeta Olympus, l'equipaggio trova la dea che presto diviene Satana e il gruppo riesce solo per un soffio a scappare (226-229).

\section{Ibridazione dei generi}

Per quanto riguarda l'ultimo piano dell'intertestualità, il ciclo eymerichiano attinge ad un'ampia varietà di generi letterari: fantasy, science fiction, giallo, poliziesco, detective novel, mystery novel, thriller, gotico, horror, new weird, fantapolitica, romanzo di formazione, romanzo neostorico. Secondo l'autore del ciclo eymerichiano la letteratura fantastica «debba superare le etichette e contaminarsi, se vuole sopravvivere» e effettuare la sua missione (Tosoni, 2002). Evangelisti crede che la fantasy e la science fiction abbiano il potere di fortificare lo spirito umano contro le crisi quali la guerra, la fine del mondo o la repressione politica e militare. «Occorre una narrativa massimalista, autoconsapevole, che inquieti e non consoli. La fantascienza lo era. Può tornare a esserlo» (Evangelisti, 2001: 121)2. Spinto da questa sua convinzione, lo scrittore,

${ }^{2}$ L'impegno sociale e morale della narrativa fantastica contemporanea auspicato da Evangelisti trasgredisce infatti il quadro definitorio della narrativa postmoderna, il che permette di collocare il filone fantastico nello spazio della narrativa post-postmoderna o ipermoderna. «Se allora il postmoderno si è pensato come l'epoca della fine della storia e dei conflitti, in questa nuova fase la storia si è rimessa in moto, i conflitti prendono di nuovo a manifestarsi, l'attrito tra vita intelettuale e assetti politico-economici è tornato a essere produttivo. [...] [Gli autori 
nel suo ciclo narrativo, mette in relazione l'elemento science fiction con quello fantasy. La tensione tra questi due filoni e le interpretazioni da loro implicate dà origine ad un forte dinamismo dell'opera e ad una creazione particolare del protagonista. Dalle tensioni tra i generi nasce il carattere antieroico di Eymerich, nel quale sta la sua originalità. L'introduzione dell'antieroe e la sua significativa psicologizzazione danno origine a tanti effetti che postmodernizzano la fantasy classica. È grazie all'elemento science fiction che Eymerich è un soggetto atto a trasformare il suo mondo ed a controllarlo. Non è soltanto un personaggio statico e immutabile con il sistema di valori inflessibile che prova solo a riparare l'ordine già stabilito nel mondo, come spesso avviene nella fantasy (TRĘBICKI, 2007: 141). Lucido di mente, perfettamente padrone delle proprie facoltà, consapevole della propria condotta, Eymerich scopre l'illusorietà e la finzionalità dell'ordine sociale, rivela la sua instabilità e il falso. Il protagonista costruisce il proprio sistema assiologico nello scontro con un mondo mutevole e privo di fondamenti forti e sopratutto nello scontro con gli altri uomini spesso da lui considerati come deboli e facilmente influenzabili e perciò degni di disprezzo (Stasiewicz, 2016: 61). Dal punto di vista della sua funzione attanziale, l'inquisitore è indubbiamente un protagonista attivo, lungi dall'essere subordinato alla trama, e diventa un ispiratore continuamente attivo degli eventi. La trama diventa così il risultato delle sue aspirazioni. È qui che nasce la riflessione del lettore sull'ideologia e sulla teologia che si manifestano nelle azioni di Eymerich. Evangelisti stabilisce un'interazione lettore-protagonista in cui il lettore viene spinto a costruirsi il giudizio sempre più negativo del protagonista, riconoscendo gli aspetti negativi del sistema dei valori rappresentato da Eymerich. Senza dubbio l'inquisitore è un eroe negativo, ma nello stesso tempo rimane affascinante ai lettori, probabilmente per il motivo che si caratterizza per il carisma, il coraggio e l'idealismo, anche se le idee a cui attinge sono definite da lui stesso.

Ma forse l'ibridazione più importante del ciclo eymerichiano si svolge non tra i generi di fantasy e science fiction, ma tra fantasy e romanzo storico, visto che sia la storia che la letteratura forniscono degli intertesti ad Evangelisti. Il ciclo adotta la forma della serie di tanti volumi e tanti fili narrativi ed introduce un vasto panorama di avvenimenti e personaggi storici, mescolando questi veri con quelli finzionali. L'effetto è un grandioso progetto intertestuale e plurigenerico della finzione metastorica nel quale Inquisitore Eymerich si avvicina al romanzo neostorico, come viene chiamato il romanzo storico postmoderno in Italia (SerkowsKa, 2003, 2012). Evangelisti attribuisce un peso notevole alla fedeltà della ricostruzione storica, modellando il mondo rappresentato sulle localizzazioni geografiche specifiche e sui periodi storici concreti. Le letture preparatorie

ipermoderni] affidano alla letteratura un compito di verità, che ha senso solo se l'opera sta dentro e davanti a un mondo che non può esaurire e che le oppone resistenza» (DonnARumma, 2014: $100,160)$. 
gli servono alla documentazione per personaggi, vicende e ambientazioni, ma anche alla costruzione del discorso critico generale, fornendogli argomenti per la riflessione sulla storia del potere ed anche sulla storia dell'uomo moderno ed il suo futuro (Sebastiani, 2018: 129). Sembra che l'autore sia affascinato principalmente dal romanzo storico e la narrazione postmoderna gli serva ad imporre un'interpretazione del testo diversa che nel caso di un classico romanzo storico. L'ascrizione del ciclo al genere fantastico determina la sua interpretazione e ricezione da parte del pubblico; se questo ciclo fosse stato scritto come un romanzo storico solo con gli elementi surreali e onirici, il lettore penserebbe che il romanzo riguarda solo la lotta per il potere nella Spagna trecentesca. Ma grazie ad essere narrato nella stilistica fantasy contaminata da altri generi, il romanzo ottiene la dimensione universale, come un mito che racconta la storia del potere che si può svolgere sempre ed ovunque.

Dato che il presente, il passato e il futuro sono inevitabilmente testualizzati e considerata la complessità degli eventi, la narrativizzazione della storia con l'aggiunta di elementi fantastici dimostra di essere un procedimento necessario per comprendere e acquisire la storia (Hutcheon, 1991: 221). Come Evangelisti stesso scrive, gli eventi sono passati, ma la psicologia che utilizza è moderna. Eymerich di Evangelisti condivide con il suo prototipo storico il nome, i dati anagrafici, l'istruzione, la produzione saggistica, il ruolo istituzionale e la posizione politica di appoggio alla chiesa avignonese, ma non imita il suo modo di parlare, né adotta il modo di pensare di quell'epoca. Evangelisti «si sente totalmente libero [...] di inventare per i suoi personaggi monologhi interiori, di attribuire loro sentimenti, intenzioni o motivazioni» (AlTER, 1990: 51-52). Circonda il personaggio principale con personaggi storici realmente esistiti (come re, papi, vescovi, nobili, letterati, alchimisti, fisici, matematici, biologi, psicoanalisti) ed anche con «personaggi inventati, destinati a servire da contrasto, da specchio, da ostacolo, o da aiuto nella sua crescita» (51-52). A ben vedere, una volta affiancati alle figure inventate, i personaggi storici anche diventano finzionali. L'autore mette la realtà e la creazione fantastica in relazione, rendendole dipendenti l'una dall'altra; nasce così un caso peculiare dell'ucronia, cioè di storia alternativa. Il mondo apparentemente autonomo e chiuso dentro i suoi confini spaziali e temporali (elemento fantasy) si apre con la comparsa delle astronavi psitroniche capaci di viaggiare nel tempo e con l'invenzione di energia psitronica nel futuro (elemento science fiction) responsabile dei fenomeni paranormali accaduti nel passato (effetto postmodernizzante). In questo senso l'autore diverge dalla verità storica, però Evangelisti sembra piuttosto condividere la convinzione di Marta Morazzoni che la verità storica si verifica solo nella finzione e che gli eventi passati possono essere interpretati, ma mai fedelmente rappresentati (MoRAZzonI, 1988). Questa mescolanza d'invenzione e realtà che ingloba tanti generi è la proposta di Evangelisti su come affrontare la storia e di come raccontarla. L'atto di collegare il fatto storico e la finzione attraverso i denominatori comuni di inter- 
testualità e narratività si rivela quindi come un'innovazione e l'espansione della portata della finzione (HutCHEON, 1991: 222). Quest'ucronia diventa un modo di commentare l'oggettività della storia ed è legato alla sfiducia naturale della poetica postmoderna verso la veridicità dei testi (STASIEWICZ, 2016: 182-183).

\section{Compartecipazione del lettore}

Nell'analisi narrativa di Inquisitore Eymerich dobbiamo mettere in rilievo che oltre al dialogo del testo con gli altri testi e generi e quello tra i singoli volumi del ciclo, abbiamo qui un altro tipo di dialogo - quello con il lettore. In quanto testo postmoderno, il ciclo intende suscitare la risposta del lettore, impegnandolo in un gioco continuo di ricerca dei rimandi. Cerca di creare riferimenti sia espliciti che impliciti ad altri testi e capovolge gli stereotipi ed i topoi culturali, rintracciare i quali è una fonte del piacere estetico del lettore. Nel ciclo eymerichiano abbiamo a che fare con quattro piani di coinvolgimento del lettore. Sul primo piano il lettore deve risolvere con Eymerich degli enigmi e giustificare le situazioni anomale che l'inquisitore incontra. Però, c'è un compito ancora più impegnativo per il lettore, quello di ricostruire il discorso che sottende a tutto il ciclo, scoprendo le connessioni tra i testi. Il ciclo Inquisitore Eymerich è frammentato in narrazioni autoconclusive all'interno di una continuità narrativa, ma con un ordine editoriale che non coincide con la cronologia degli eventi. Ci sono argomenti che sono solo nominati in un romanzo per essere poi sviluppati nei successivi, come ad esempio i giganti introdotti in Mater terribilis attraverso una sola parola che appaiono poi in La luce di Orione come la prole del demone evocato per generarli per sconfiggere un nemico ed anche in Rex tremendae maiestatis come le allucinazioni generate da un sistema cibernetico derivate dalle leggende siciliane. $\mathrm{Ci}$ sono anche gli eventi che sono logiche conseguenze delle situazioni descritte altrove come per esempio il conflitto con Pietro IV in Mistero dell'inquisitore Eymerich che dà origine a quello tra il re e l'Inquisizione in Picatrix e in Castello di Eymerich, sospeso in Rex tremendae maiestatis e poi di nuovo presente in Fantasma di Eymerich quando l'inquisitore viene imprigionato. Accade anche che gli stessi concetti siano riproposti in diversi volumi come quello dell'abuso di potere sull'esempio di Genovesi e Veneziani nell'Impero Romano d'Oriente e dei baroni e il popolo siciliano in La luce di Orione e Rex tremendae maiestatis. Quindi il lettore è invitato a ricostruire l'intera storia attraverso una riorganizzazione logica e cronologica degli elementi narrativi ricorrenti che danno coesione e coerenza al discorso. Il terzo piano del coinvolgimento del lettore consiste nel distinguere tra gli eventi realmente accaduti e le vicende inventate nella realtà storica narrata ucronicamente. 
Però, per quanto riguarda il dialogo con il lettore, l'aspetto che balza all'occhio più degli altri, è la rottura della distanza tra il testo e il lettore. Il ciclo eymerichiano stabilisce una dinamica relazione partecipativa con il lettore, in cui quest'ultimo diventa creatore e rielaboratore attivo che non si limita a leggere, ma interagisce, impadronendosi del personaggio dell'inquisitore attraverso la produzione fan fiction sollecitata da Evangelisti stesso. Al solito il lettore implicito non viene identificato con il lettore reale. Qui però, grazie al ricorso alla narrazione postmoderna, il lettore implicito si fonde con il lettore reale che utilizza la sua conoscenza letteraria, storica e culturale per continuare a raccontare la storia del protagonista al di là dei limiti del libro di Evangelisti. La copiosità delle narrazioni apocrife rende difficile la loro individuazione e catalogazione esaustiva, ma certo è che tra il 2003 e il 2014 sono stati pubblicati 28 racconti editi on line su siti specializzati di fan fiction, su blog diversi, sulla rivista Carmilla on line e nelle due antologie nate da concorsi letterari: I segreti di Eymerich (Solid 2003) e Scimmiette di mare (Nero Press 2013). Inoltre nel 2005 è stato pubblicato il romanzo La potenza di Eymerich (Bacchilega 2005), il risultato di una sfida in rete lanciata dal collettivo letterario Kai Zen e realizzata da un sodalizio di autori chiamato Emerson Krott.

Il personaggio dell'Inquisitore Eymerich è diventato quasi indipendente dal suo creatore, un fatto che molti scrittori non accetterebbero mai. Nel caso di Eymerich, la vitalità del personaggio scaturisce dal fatto che grazie proprio ad Internet, Evangelisti era in grado di tenersi in contatto con i suoi fan più fedeli. Lo scrittore è consapevole che la nascita della Rete ha generato nuove opportunità nell'ambito della narrazione e innovazioni profonde nel bisogno stesso di praticare lo storytelling. Internet, un autentico incubatore di pratiche narrative, ha cambiato le dinamiche dello scambio letterario: ora l'autore può leggere ciò che scrive il lettore. La qualità peculiare della lettura e della scrittura su Internet è che si scelgono ordini differenti da quello cronologico. Allora il fatto che il ciclo eymerichiano non seguiva un criterio cronologico ha soddisfatto i gusti degli internauti e gli ha fornito i punti della trama aperti da riempire con narrazioni fan fiction. La fan fiction ha contribuito all'espansione del ciclo seguendo tutte e tre le linee guida individuate da Doležel per la creazione di un universo narrativo, ossia ha dato vita a mondi paralleli e polemici, ha colmato lacune con prequel o sequel e ha costruito nuove versioni del protomondo, ridefinendone la struttura e reideando la storia (DoležEL, 1999: 207-208). Quindi, nel ciclo non ci sono solo tre dialoghi intertestuali, ma c'è infatti anche il quarto - quello dei testi canonici con i testi apocrifici di fan fiction, il che si presta ad un tipo particolare dell'interestualità, la cosiddetta transfinzionalità, dove gli apocrifi condividono personaggi e ambientazioni del testo primario come se esistessero indipendentemente (CALABRESE, 2010: 206).

Secondo Evangelisti la proliferazione di fan fiction non indebolisce il ciclo originario, al contrario, la dilata. «Chiunque scriva deve tenere presente il nuovo 
assetto mediatico che si sta proliferando. L'opera cui ha dato vita, nell'immediato futuro, non sarà soltanto sua. I personaggi che ha creato potranno finire in mani altrui» (EvangelisTi, 2005: 8). Curioso è che tenere la letteratura sotto il controllo sia stato uno dei primi scopi dell'Inquisizione e l'inquisitore Eymerich stesso è caduto vittima alla moltiplicazione rapida ed incontrollata dei testi apocrifici che probabilmente lui stesso escluderebbe dal canone come non autentici. E il suo creatore, anzi, non solo non autorizza i fan fiction proliferanti, ma anche ogni rappresentazione dell'inquisitore con il pretesto di rinarrare o analizzare gli eventi del passato o del presente oppure prospettare il futuro, la sente come sua (8). Però, da questo ampliamento enorme del bacino dei destinatari, «sia in qualità di lettori passivi che di creatori (o anche rielaboratori) attivi», deriva una responsabilità di «passare ad argomenti di interesse generale» (8). Sia la scrittura di Evangelisti sia quella da lui sollecitata sono al tempo stesso popolari e impegnate offrendo uno «sguardo lucido e critico sulla realtà» (Evangelisti in: Tosoni, 2002). Secondo Evangelisti, la letteratura di genere è «meglio attrezzata per adottarlo di quanto non lo sia la letteratura cosidetta alta, che da un pezzo sembra avere completamente rinunciato ad analizzare il mondo che ci circonda» (Evangelisti in: Tosoni, 2002). L'auspicio di Evangelisti è quindi che «grazie a Internet, cento Eymerich sboccino, cento visioni critiche del presente gareggino» (Evangelisti, 2005: 9). E infatti sono apparsi anche altri tipi di narrazioni che riutilizzano il personaggio dell'inquisitore. La significativa espansione del ciclo è realizzata attraverso opere musicali (il libretto di Tanit e il testo di Terrible Mother), videogiochi (Nicolas Eymerich, Inquisitore: La Peste, 2012), fumetti (I cristalli di Eymerich, 2003; La dea: Nicolas Eymerich inquisitore, 2003; Il corpo e il sangue: Nicolas Eymerich, 2003), sceneggiature (La furia di Eymerich, 2001) e radiodrammi (La scala per l'inferno, 1998; Il castello di Eymerich, 2000; La furia di Eymerich, 2001; L'inquisitore e i portatori di luce, 2011). A ben vedere, Evangelisti tende a uscire sempre fuori dal testo, visto che Inquisitore Eymerich attinge ai media diversi e coinvolge i lettori extradiegeticamente invitandoli alla scrittura e si sviluppa coestensivamente attraverso le continuazioni finzionali parallele.

\section{Conclusioni}

Secondo Umberto Eco, ogni epoca ha il proprio postmoderno che consiste nel rivisitare tutto ciò che quest'epoca ha prodotto, scontrandolo con i testi scritti ancora prima (Eco, 1983: 21). Come è stato messo in evidenza, lo sviluppo della letteratura fantasy in Italia ha conosciuto il suo apice proprio nel postmodernismo, ma si deve tener presente che la letteratura fantastica è dal principio sin- 
cretica e derivante da diverse fonti, allora la fase postmoderna è nient'altro che una continuazione naturale dell'evoluzione del genere e definitivamente non un segno dell'esaurimento della letteratura, come lo comprova la narrativa del decano della fantasy italiana, Valerio Evangelisti. Gli elementi postmoderni come la dinamica dialogicità e la relazionalità sono ben visibili nel ciclo eymerichiano: tutto dialoga, il ciclo con gli altri testi di cultura, un volume con gli altri volumi ed anche i piani sovratemporali l'uno con l'altro, poi nella trama la materia interagisce con la mente e l'autore interagisce con il lettore. Dal ciclo emerge che l'elemento fantastico dovrebbe condividere la sfiducia della scienza moderna nelle soluzioni globali e nelle istituzioni religiose ed entrare in collisione con vari sistemi, stili e filoni letterari e conoscenze provenienti da vari campi. Lautore intraprende una grande impresa di finzione metastorica in cui spazia tra i generi dalla fantasy alla science fiction e, sovrapponendo i piani spazio-temporali, crea una totalizzante analisi della storia umana con l'accento sulla fenomenologia del potere. Invita il lettore ad un gioco intelettuale e poi stabilisce una dinamica relazione partecipativa con il lettore, in cui quest'ultimo diventa un creatore e rielaboratore attivo attraverso la produzione fan fiction. Sfruttando la capacità del fantasy di penetrare in media diversi ed intrecciando i generi letterari e le narrazioni di media diversi, Evangelisti crea un nuovo tipo del testo fantastico impegnato, la post-fantasy, di cui ha già alcuni sostenitori come Vanni Santoni, Francesco Dimitri e Francesco Barbi. La post-fantasy supera la barriera tra il fantastico per letterati e il fantastico di genere, essendo infatti non una forma ibrida, ma una concezione totalmente nuova della narrazione postmoderna.

\section{Bibliografia}

Alter, Robert, 1990: L'arte della narrativa biblica. Brescia, Queriniana.

CAlABrese, Stefano, 2010: "Forme contemporanee". In: La comunicazione narrativa. Dalla letteratura alla quotinidianità. Milano-Torino, Mondadori, pp. 198-239.

Ceserani, Remo, 1997: Raccontare il postmoderno. Torino, Bollati Boringhieri.

Doležel, Lubomir, 1999: Heterocosmica. Fiction e mondi possibili. Milano, Bompiani.

Donnarumma, Raffaele, 2014: Ipermodernità. Dove va la narrativa contemporanea. Bologna, il Mulino.

Eco, Umberto, 1983: "Postille a Il nome della rosa". Alfabeta, no 49, pp. 19-22.

Evangelisti, Valerio, 1994: Nicolas Eymerich, inquisitore. Milano, Mondadori.

Evangelisti, Valerio, 2001: "Una narrativa adeguata ai tempi". In: Alla periferia di Alphaville. Napoli, L'Ancora del Mediterraneo, pp. 113-121.

Evangelisti, Valerio, 2005: "Introduzione". In: La potenza di Eymerich. Kai Zen e Emerson Krott. Imola, Bacchilega, pp. 5-9.

HutCHEON, Linda, 1991: „Historiograficzna metapowieść: parodia i intertekstualność historii”. Pamiętnik Literacki, no 82/4, pp. 216-229. 
LiPPI, Giuseppe, 2000: "Un universo immaginario del pensiero: Valerio Evangelisti (Introduzione)". In: Nicolas Eymerich, inquisitore. Milano, Classici Urania (Mondadori), no 278, pp. 7-15.

Morazzoni, Marta, 1988: L'invenzione della verità. Milano, Longanesi.

Sebastiani, Alberto, 2015: "La fan fiction e la politica. Il caso Eymerich e la critica alla Chiesa cattolica". Between, Vol. 5, no 10, <https:/ojs.unica.it/index.php/between/article/ view/1582/1831>. Data dell'ultima consultazione: 03.06.2020.

Sebastiani, Alberto, 2018: Nicolas Eymerich. Il lettore e l'immaginario in Valerio Evangelisti. Bologna, Odoya.

SERKowsKa, Hanna, 2003: „O postmodernizmie we Włoszech”. Teksty Drugie: teoria literatury, krytyka, interpretacja, no 6 (84), pp. 179-197.

Serkowska, Hanna, 2012: Dopo il romanzo storico. La storia nella letteratura italiana del '900. Pesaro, Edizioni Metauro.

Somigli, Luca, 2006: "Guerre senza fine: 'scontri di civilità' passati e futuri nella prima narrativa di Valerio Evangelisti”. Narrativa, no 28, pp. 193-211.

Stasiewicz, Piotr, 2016: Między światami. Intertekstualność i postmodernizm w literaturze fantasy. Białystok, Wydawnictwo Uniwersytetu w Białymstoku.

Tosoni, Simone, 2002: "Valerio Evangelisti. Diario di un inquisitore". Trax, 18 ottobre, $<$ http:// www.trax.it/valerio_evangelisti.htm>. [Data dell'ultima consultazione: 03.06.2020].

TRenTI, Lorenzo, 2019: "Eymerich si scrive da sinistra". Fantascienza.com, 16 gennaio, <https:// www.fantascienza.com/24312/eymerich-si-scrive-da-sinistra>. [Data dell'ultima consultazione: 03.06.2020].

TRĘBICKI, Grzegorz, 2007: Fantasy. Ewolucja gatunku. Kraków, Universitas.

\section{Nota biobibliografica}

Patrycja Przelucka - laureata in filologia inglese e filologia italiana presso l'Università della Slesia a Katowice. I suoi interessi sono incentrati sulla letteratura fantastica contemporanea, specialmente il romanzo fantastico italiano dopo il 1970. Nel 2017 per la casa editrice SQN ha tradotto in polacco i seguenti libri: Deep: Freediving, Renegade Science, and what the Ocean Tells Us about Ourselves di James Nestor e Fedegraphica: A Graphic Biography of the Genius of Roger Federer di Mark Hodgkinson. Dal 2019 esegue il dottorato presso la Scuola Dottorale dell’Università della Slesia. Dal 2017 al 2019 ha insegnato la lingua e cultura italiana presso l'Istituto di Lingue Romanze e Traduttologia dell'Università della Slesia.

patrycja.przelucka@us.edu.pl 